\title{
Theta Oscillations Reflect the Dynamics of Interference in Episodic Memory Retrieval
}

\author{
Tobias Staudigl, Simon Hanslmayr, and Karl-Heinz T. Bäuml \\ Department of Experimental Psychology, Regensburg University, 93053 Regensburg, Germany
}

Selectively retrieving episodic information from a cue often induces interference from related episodes. To promote successful retrieval of the target episode, such interference is resolved by inhibition, causing retrieval-induced forgetting of the related but irrelevant information. Passively studying the episodic information again (reexposure) does not show this effect. This study examined the hypothesis that brain oscillations in the theta band $(5-9 \mathrm{~Hz})$ reflect the dynamics of interference in selective memory retrieval, analyzing EEG data from 24 healthy human subjects ( 21 women, 3 men). High versus low levels of interference were investigated by comparing the effects of selective retrieval with the effects of reexposure of material, with the former, but not the latter, inducing interference. Moreover, we analyzed repeated cycles of selective retrieval and reexposure, assuming that interference is reduced by inhibition across retrieval cycles, but not across reexposure cycles. We found greater theta band activity in selective retrieval than in reexposure, and a reduction in theta amplitude from the first to the second cycle of retrieval predicting the amount of retrieval-induced forgetting; the sources of theta amplitude reduction across retrieval cycles were located in the anterior cingulate cortex. No difference in theta activity was found across repeated cycles of reexposure. The results suggest that higher levels of interference in episodic memory are indexed by more theta band activity, and that successful interference resolution via inhibition causes a reduction in theta amplitude. Thus, theta band activity can serve as a neural marker of the dynamics of interference in selective episodic retrieval.

\section{Introduction}

In present-day information society, successfully dealing with interfering information is essential. For instance, searching for a specific information on the internet provides a huge amount of results, some being relevant and others irrelevant. Similarly, searching for past episodes in memory activates relevant and irrelevant memories. The competition among them (termed interference) needs to be resolved to promote retrieval of the relevant information (Anderson and Neely, 1996; Bäuml, 2008). Here, we investigate whether the dynamics of such interference in episodic memory retrieval are reflected in theta oscillatory activity.

Using the retrieval-practice paradigm (Anderson et al., 1994), previous studies repeatedly demonstrated that selective memory retrieval induces interference from related information (for review, see Anderson, 2003, or Bäuml et al., 2010). After studying a categorized item list (e.g., FRUIT-Orange, FRUIT-Banana, DRINK-Vodka), attempts to retrieve a specific target item (FRUIT-Or_) induce interference from related, not-to-beretrieved items (Banana), which must be resolved to promote selection of the target information. Theoretically, interference resolution is achieved via inhibition, which suppresses the interfering information's memory representation. The behavioral ef-

\footnotetext{
Received Feb. 5, 2010; revised June 22, 2010; accepted July 12, 2010.

We thank M. Wimber and three anonymous reviewers for helpful comments on a previous version of this manuscript, and L. Abel and S. Schwaiger for their help with data acquisition.

Correspondence should be addressed to Karl-Heinz T. Bäuml, Department of Experimental Psychology, Regensburg University, Universitätsstrasse 31, 93053 Regensburg, Germany. E-mail: karl-heinz.baeuml@ psychologie.uni-regensburg.de.

DOI:10.1523/JNEUROSCI.0637-10.2010

Copyright $\odot 2010$ the authors $\quad 0270-6474 / 10 / 3011356-07 \$ 15.00 / 0$
}

fect of this, i.e., the subsequent forgetting of the interfering information, is termed retrieval-induced forgetting (Anderson and Spellman, 1995; Spitzer and Bäuml, 2007). fMRI findings suggest that interference and its resolution in selective memory retrieval are related to initial activation and subsequent downregulation of activation in anterior cingulate cortex (ACC) and lateral prefrontal areas (Kuhl et al., 2007, 2008; Wimber et al., 2008, 2009).

Previous EEG studies established a link between cognitive interference and theta oscillations. For instance, in classic interference paradigms (e.g., Stroop task, Flanker task), prefrontal theta oscillations varied with interference level (Hanslmayr et al., 2008; Cavanagh et al., 2009). These and other studies indicated that midfrontal theta oscillatory activity is generated in the ACC (Gevins et al., 1997; Hanslmayr et al., 2008), complementing imaging studies pointing to the ACC as the neural source for interference detection (Botvinick et al., 2001). In the memory domain, a relation between theta amplitude and size of memory set was found in working memory tasks (Mecklinger et al., 1992; Jensen and Tesche, 2002), but evidence that theta oscillations could reflect the dynamics of interference in episodic memory is still lacking.

Using a modification of the retrieval-practice paradigm, this study examines the hypothesis that theta oscillations reflect interference in selective memory retrieval. Because retrieval, but not reexposure, of previously studied material induces interference in episodic memory (Anderson et al., 2000; Bäuml and Aslan, 2004), we examined whether selective retrieval, but not reexposure, of material affects oscillatory theta activity. Examining the impact of inhibition, we analyzed retrieval and reexposure across 


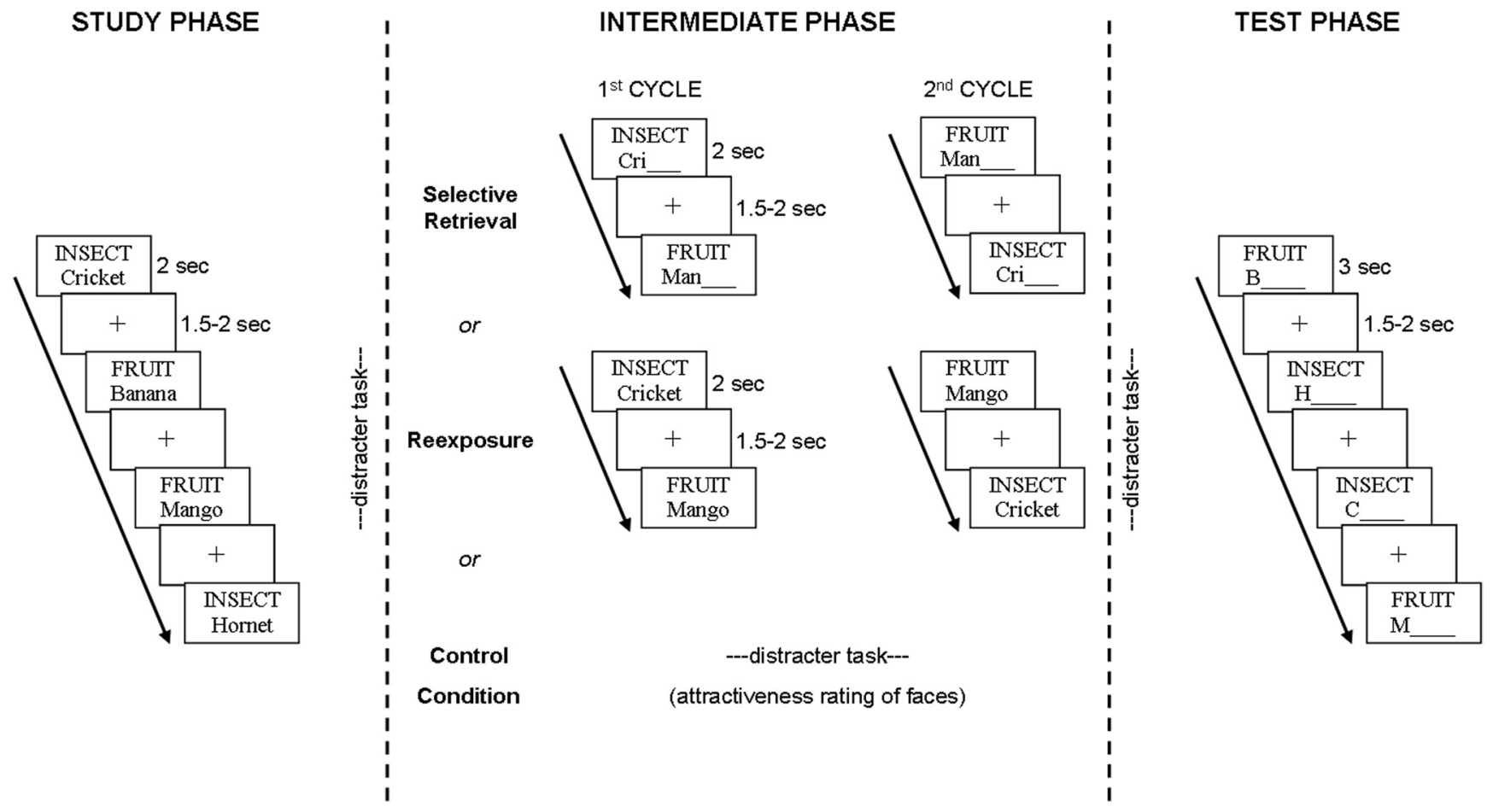

Figure 1. The experimental procedure as used in the present study. The experiment consisted of three blocks, each block comprising a study phase, an intermediate phase, and a test phase. In the study phase of each block, items were studied together with their category cue. In the test phase of each block, all of the items were tested using a cued recall test. The intermediate phase varied with block: in the selective retrieval block, a subset of the previously studied items was retrieved on two consecutive cycles; in the reexposure block, the same subset of items was restudied on two consecutive cycles; in the control block, no practice took place and subjects conducted a distracter task instead. EEG was recorded during the intermediate phase of the selective retrieval and the reexposure block.

repeated practice cycles, expecting that theta activity is reduced across retrieval cycles (Kuhl et al., 2007), but is largely unaffected across reexposure cycles. Particularly, we hypothesized that the sources of the theta band activity are localized in the ACC. Finally, grouping participants according to their behavioral effects of inhibition, i.e., the amount of retrieval-induced forgetting, we expected differences in theta activity between participants to reflect the different degrees of retrieval-induced forgetting.

\section{Materials and Methods}

\section{Subjects}

Twenty-seven adults served as subjects in the experiment. Three of them were excluded from data analysis, because their EEG was heavily contaminated by movement artifacts. The remaining 24 subjects (19-33 years old, 21 women, 3 men) participated voluntarily in the experiment, had normal or corrected to normal vision, had German as their native language, and reported no history of neurological disease. Subjects received course credits or $25 €$ for participation.

\section{Materials}

Stimuli were 216 German nouns from 36 semantic categories, drawn from several published norms (Mannhaupt, 1983; Scheithe and Bäuml, 1995). Each category consisted of six items with unique first letters with respect to their category. Within a category, four items were lowfrequency exemplars within their category (mean rank $=25.1, \mathrm{SD}=$ 7.2); they were practiced in the intermediate phase of the reexposure and the selective retrieval condition, and are referred to as "to-be-practiced items." Two items within each category were high-frequency exemplars within their category (mean rank $=7.8, \mathrm{SD}=1.5$ ); they were not practiced, and are referred to as "unpracticed items." Previous work has shown that high-frequency items of a category may be more likely than low-frequency items to be subject to retrieval-induced forgetting (Anderson et al., 1994; Bäuml, 1998).

\section{Design}

An outline of the experimental design can be seen in Figure 1. The experiment consisted of three blocks (within-subjects design), each of which comprised a study phase, an intermediate phase, and a test phase. Blocks differed in the list that was provided and the type of condition (selective retrieval vs reexposure vs control) conducted during the intermediate phase: In the selective retrieval condition, category labels and word stems of the four to-be-practiced (low-frequency) items (FRUIT-Man_) of each category were presented; subjects were instructed to retrieve the corresponding items. In the reexposure condition, category labels together with the categories' four to-be-practiced (low-frequency) items (FRUITMango) were presented intact; subjects were instructed to restudy the items to enhance their performance on the upcoming test (e.g., Bäuml and Aslan, 2004). Both practice procedures were repeated once in the intermediate phase, resulting in a first ("SR1") and second ("SR2") practice cycle in the selective retrieval condition and a first ("RE1") and second ("RE2") practice cycle in the reexposure condition. In the control condition, no practice of studied items took place, and subjects performed a distracter task instead. This design created practiced (low frequency) and unpracticed (high frequency) items for both the selective retrieval and the reexposure condition. Although there was no practice in the control condition, control items were split according to their word frequency to match practiced and unpracticed items. Accordingly, the beneficial effect (enhancement) of practice was defined as the difference between low-frequency items in the control condition and practiced (low-frequency) items in either the selective retrieval or the reexposure condition. The detrimental effect (forgetting) was defined as the difference between high-frequency control items and unpracticed (highfrequency) items, in either the selective retrieval or the reexposure condition. The assignment of list to condition was counterbalanced across subjects, and the order of blocks was randomized.

\section{Procedure}

Subjects were tested individually in a quiet surrounding, seated in front of a 15 inch computer screen $(70 \mathrm{~Hz}$ refresh rate). 
Study phase. Each of the three conditions started with a study phase, in which one list, consisting of 72 items from 12 different categories (e.g., FRUIT-Mango), was presented. The items of a list were presented sequentially in random order. A trial in the study phase started with a fixation cross with variable duration $(1500-2000 \mathrm{~ms})$. Thereafter the stimulus word together with its category label was presented for $2000 \mathrm{~ms}$. After the study phase, the subjects performed a distracter task for $30 \mathrm{~s}$, during which they were asked to rate pictures of famous and not famous people according to their attractiveness.

Intermediate phase. In the selective retrieval condition, subjects were asked to retrieve the respective memory item, which was cued by its category label and its unique word stem. Following Johansson et al. (2007), subjects were asked to covertly retrieve the to-be-practiced items during selective retrieval, to avoid EEG artifacts elicited by speech. In the reexposure condition, to-be-practiced items were presented intact together with their category label. Subjects were asked to (quietly) restudy the items for the final recall test. In both the retrieval and the reexposure condition, a trial consisted of a fixation cross that was shown with variable duration (1500-2000 ms). Thereafter the memory item (reexposure condition) or the item's word stem (retrieval condition) was presented for $2000 \mathrm{~ms}$ together with the item's category label. The items' order in the intermediate phase was block randomized, with four blocks, each containing one low-frequency item from each of the lists' categories. Both practice procedures were repeated once in the intermediate phase and ended by another distracter task (attractiveness rating) of $30 \mathrm{~s}$. In the control condition, subjects performed a distracter task (attractiveness rating) rather than retrieving or relearning any items. Duration of the intermediate phase was held constant over conditions.

Test phase. After the intermediate phase, a cued recall test was performed in which all items were tested. A fixation cross with variable duration (1500-2000 ms) was presented before the memory item's cue. The memory cue consisted of the category name together with the first letter of the item, and was shown for $3000 \mathrm{~ms}$. After that, participants were asked to respond orally with the corresponding item of the study list. Unpracticed (high-frequency) items of a category were tested first, followed by the practiced (low-frequency) items of the category. Order of categories was held constant across subjects, except for being reversed after half of the subjects. Mean recall frequency was used as dependent variable.

\section{EEG recording}

During the intermediate phase in the selective retrieval and the reexposure conditions, the EEG was recorded from $62 \mathrm{Ag} / \mathrm{AgCl}$ scalp electrodes positioned according to the extended 10-20 system. EEGs were recorded against a reference electrode placed at $\mathrm{FCz}$ and were later re-referenced against a common average reference. The vertical and the horizontal electrooculogram (EOG) were recorded from two additional channels to control for eye movements and blinks. Impedances were kept below 20 $\mathrm{k} \Omega$. A Brainamp MR + amplifier (Megis, BrainVision) was used for data acquisition. Sampling rate was $500 \mathrm{~Hz}$. Frequencies between 0.3 and 70 $\mathrm{Hz}$ with a Notch-filter at $50 \mathrm{~Hz}$ were recorded. Before data analysis, EEG data were corrected for EOG artifacts using calibration data to generate individual artifact coefficients and the algorithm implemented in the software package BESA [MEGIS Software BESA v5.1.8; see Ille et al. (2002) for details]. Remaining artifacts, due to muscle activity or poor EOG correction, were excluded by visual inspection.

\section{Analysis of oscillatory amplitude}

Time-frequency analyses in the theta band $(5-9 \mathrm{~Hz})$ were conducted using the BESA (MEGIS Software BESA v5.1.8) software package and self-written Matlab codes (The MathWorks). The EEG data were segmented into $2000 \mathrm{~ms}$ epochs (ranging from $500 \mathrm{~ms}$ preceding stimulus onset to $1500 \mathrm{~ms}$ after stimulus onset). To avoid filter artifacts at the edges of the epochs, the data were filtered in a slightly bigger time interval, but analysis was restricted to the $2000 \mathrm{~ms}$ time window. For analysis of oscillatory power, data were filtered in a frequency range of $4-20 \mathrm{~Hz}$ using BESA's time-frequency analysis module, with time-frequency resolution set to $111 \mathrm{~ms}$ and $1.99 \mathrm{~Hz}$ (full width at half maximum). Data were exported in bins of $50 \mathrm{~ms}$ and $1 \mathrm{~Hz}$.

To analyze event-related amplitude changes, the percentage of amplitude change in the theta band $(5-9 \mathrm{~Hz})$ in relation to a prestimulus baseline (set to

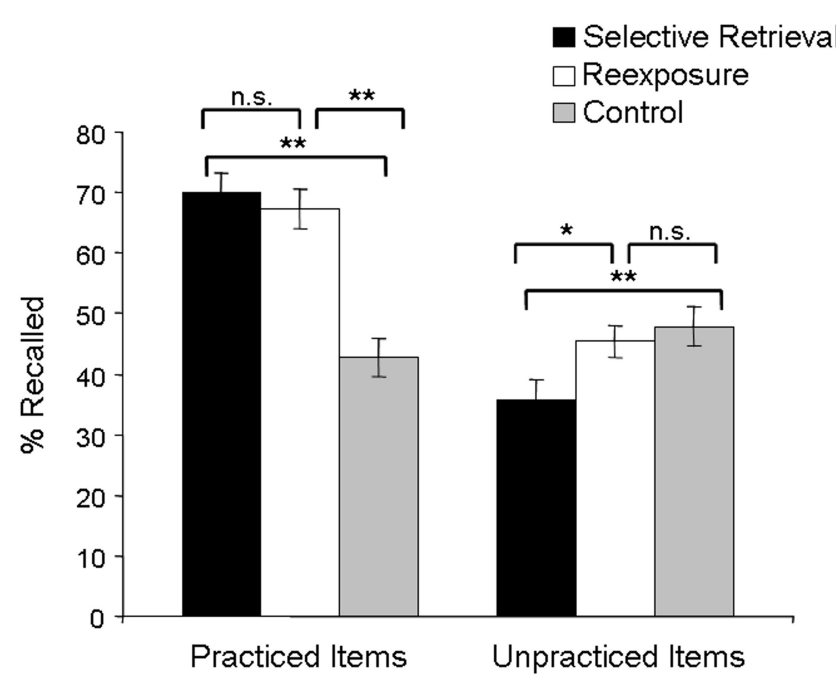

$$
\star=p<.05 ;{ }^{\star}=p<.01
$$

Figure 2. Behavioral results. Forgetting, as indicated by the difference in recall rates between unpracticed high-frequency items and high-frequency control items, is depicted on the right (error bars indicate SE). Whereas recall in the reexposure condition did not differ from recall in the control condition, less items were recalled in the selective retrieval condition than in the reexposure and the control condition. On the left, enhancement, as indicated by the difference in recall rates between practiced low-frequency items and low-frequency control items, is depicted. Recall rates did not differ between the selective retrieval and the reexposure condition, but in both conditions more items were recalled than in the control condition.

500 to $0 \mathrm{~ms}$ prior to stimulus onset) was calculated. For statistical comparisons, the data were collapsed over three consecutive time windows: T1 (0$500 \mathrm{~ms}) ;$ T2 (500-1000 ms); and T3 (1000-1500 ms).

We conducted paired comparisons (Wilcoxon signed-rank tests, twotailed) across conditions in the first cycle (SR1 vs RE1) and between the first and the second cycle for each condition (SR1 vs SR2; RE1 vs RE2). Additionally, we tested for condition-by-cycle interaction effects to investigate whether the difference between SR1 and SR2 is reliably bigger or smaller than the difference between RE1 and RE2. Time windows exhibiting significant effects were identified for each electrode position. The number of electrodes showing significant effects $(p<0.05)$ was then checked for significance by a randomization test using 2000 permutation runs. In this procedure, the two conditions were interchanged randomly for each subject and each randomization run, thereby eliminating any systematic difference between the conditions. After each randomization run, Wilcoxon signed-rank tests were calculated, returning the number of electrodes showing a significant difference between the two conditions. After 2000 permutation runs, a random distribution of the number of electrodes showing significant effects is generated. This distribution is used to determine the $p$ level of an effect, reflecting how many times a given number of significant electrodes (e.g., 8) was exceeded during the permutation runs (values higher than 8 ). Thus, the $p$ level reflects the probability that a given number of electrodes exhibiting a significant difference between two conditions is found by chance. This method is based on the algorithm described by Blair and Karniski (1993) and was already applied in several other studies from our laboratory (Hanslmayr et al., 2007, 2009).

\section{Source localization}

To localize the sources of the theta band activity, the Multiple-Source Beamformer approach was used as implemented in BESA. This method is a modified version of the linearly constrained minimum variance vector Beamformer (Gross et al., 2001). This algorithm allows to image evoked and induced oscillatory activity in a user-defined time-frequency domain. Note that, different to other source analysis methods, like e.g., dipole-fitting techniques, this approach does not require a priori knowledge of the number or location of sources. The Beamformer computes the changes of power in a poststimulus interval relative to a prestimulus 
Table 1. Number of electrodes showing significant differences in the comparisons across conditions (SR1 > RE1/SR1 < RE1), retrieval cycles (SR1 > SR2/SR1 < SR2), and reexposure cycles (RE1 $>$ RE2/RE1 $<$ RE2) and in the interaction analysis $([S R 1-S R 2]>[R E 1-R E 2] /[S R 1-S R 2]<[R E 1-R E 2])$

\begin{tabular}{lcrl}
\hline & T1 & \multicolumn{1}{l}{ T2 } & T3 \\
\hline Selective retrieval versus reexposure (1st cycle) & $12^{* *} / 0$ & $1 / 0$ & $0 / 9^{*}$ \\
First versus second cycle (selective retrieval) & $20^{* *} / 0$ & $9^{*} / 0$ & $0 / 2$ \\
First versus second cycle (reexposure) & $1 / 0$ & $0 / 1$ & $0 / 1$ \\
Interaction & $7 / 0$ & $11^{*} / 0$ & $1 / 1$ \\
\hline
\end{tabular}

${ }^{*} p_{\text {corr }}<0.05 ;{ }^{* *} p_{\text {corr }}<0.01$.

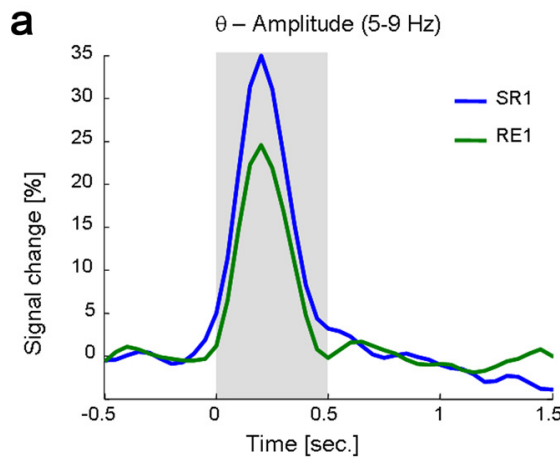

b

C

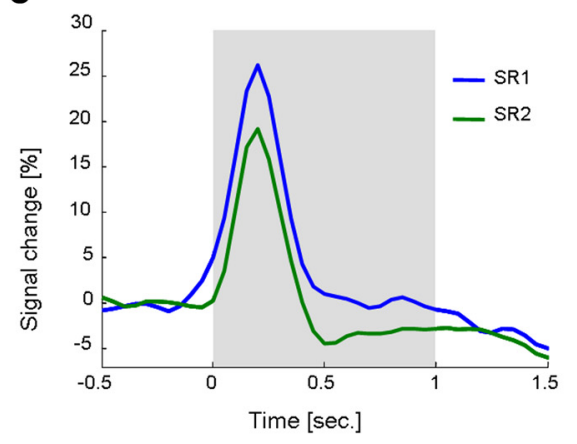

d
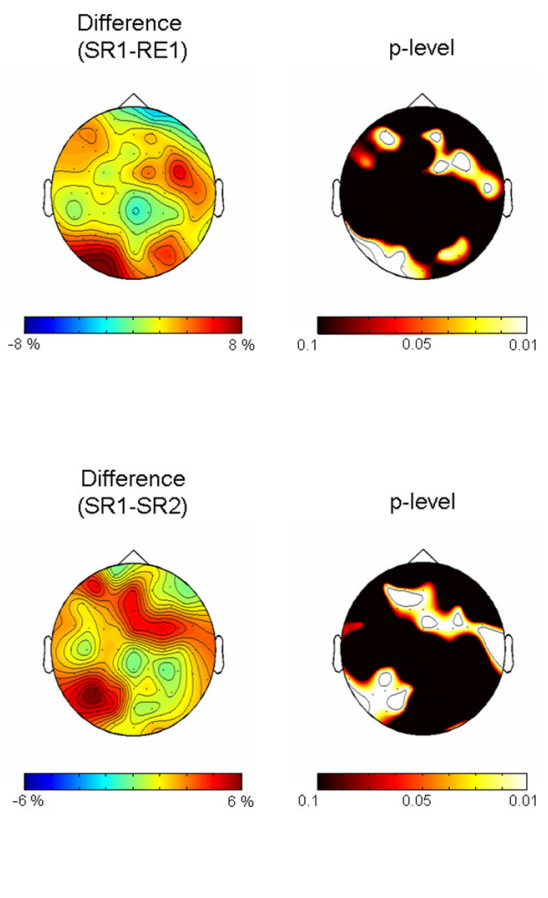

Figure 3. $\boldsymbol{a}$, The time course of theta amplitude in the first cycle of the selective retrieval (SR1) and the reexposure (RE1) condition is depicted for those electrode positions that showed a significant effect (SR1 vs RE1) during the first time window $(0-500 \mathrm{~ms})$. Time windows corresponding to adjacent topographies are colored gray. $\boldsymbol{b}$, The topography of the difference in theta amplitude in the first time window $(0-500 \mathrm{~ms})$ between the selective retrieval and the reexposure condition is shown. The right plot shows significant electrode sites, obtained by nonparametric Wilcoxon signed-rank tests. $c$, The time course of theta amplitude in the first (SR1) and second (SR2) cycle of the selective retrieval condition is shown for those electrode positions that showed a significant effect (SR1 vs SR2) during the first and second time window (0-1000 ms). Time windows corresponding to adjacent topographies are colored gray. $\boldsymbol{d}$, The topography of the difference in theta amplitude between the first and second cycle of the selective retrieval condition, collapsed over the first and second time window $(0-1000 \mathrm{~ms})$, is shown. The right plot shows significant electrode sites, obtained by nonparametric Wilcoxon signed-rank tests.

baseline (set to 500 to $0 \mathrm{~ms}$ prior to stimulus onset), transforming data into standard MNI space (Montreal Neurological Institute, Montreal, Quebec, Canada). Beamformer source analysis was performed for each cycle in the selective retrieval condition separately (SR1 and SR2), but only for those time-frequency windows that exhibited significant effects in the interaction analysis on the scalp electrode level. Differences in power in the theta band $(5-9 \mathrm{~Hz})$ between SR1 and SR2 were examined by Wilcoxon tests. Analysis and plotting of the results was done using the Matlab toolbox Fieldtrip (can be downloaded at http://www.ru.nl/fcdonders/fieldtrip) and selfwritten Matlab codes (can be requested from the second author).

\section{Results}

\section{Behavioral results}

Forgetting

Mean recall performance for unpracticed items was 35.6\% ( $\mathrm{SE}=$ $3.6)$ in the selective retrieval condition, $45.3 \%(\mathrm{SE}=2.6 \%)$ in the reexposure condition, and $47.8 \%(\mathrm{SE}=3.3)$ in the control con- dition (Fig. 2). A one-way ANOVA for the unpracticed items yielded a significant main effect for condition (selective retrieval, reexposure, control $)\left(F_{(2,46)}=4.94, p<0.05\right)$. Recall performance for unpracticed items in the selective retrieval condidiffered significantly from the control condition $\left(t_{(23)}=\right.$ , $p<0.01)$ and the reexposure condition $\left(t_{(23)}=2.55, p<\right.$ .05). In the reexposure condition, subjects did not recall significantly less unpracticed items than in the control condition $\left(t_{(23)}=0.54, p=0.59\right)$.

\section{Enhancement}

Mean recall performance for practiced items was $70.0 \%(\mathrm{SE}=3.0)$ in the selective retrieval condition, $67.1 \%(\mathrm{SE}=3.3)$ in the reexposure condition, and $42.8 \%$ $(\mathrm{SE}=3.1)$ in the control condition (Fig. 2). A one-way ANOVA for the practiced items yielded a significant main effect for condition (selective retrieval, reexposure, control $),\left(F_{(2,46)}=95.2, p<0.001\right)$. Recall rates for practiced items in the selective retrieval condition and the reexposure condition did not differ $\left(t_{(23)}=1.45, p=\right.$ $0.16)$, but in both conditions, subjects recalled more practiced items than in the control condition $\left(t_{(23)}>9.5, p<0.001\right.$, for both paired comparisons). Enhancement was not significantly correlated with forgetting $(r=-0.15, p=0.49)$.

\section{EEG results}

An overview of the statistical results of theta band amplitudes is shown in Table 1.

Selective retrieval versus reexposure

Comparing the first cycle of the selective retrieval (SR1) with the first cycle of the reexposure (RE1) condition, statistical analyses revealed a difference in the first time window in the theta band $(5-9 \mathrm{~Hz}$, $\left.0-500 \mathrm{~ms}, p_{\text {corr }}<0.01\right)$. Figure $3 a$ shows the larger increase in theta amplitude in SR1 than RE1. The difference was mainly located over midfrontal and right frontal and left parietal electrode sites (Fig. $3 b$ ). Whereas there was no difference between conditions in the second time window (500-1000 ms), a significant effect was observed in the third time window (1000$1500 \mathrm{~ms}$ ), where theta amplitude over central and right parietal electrodes was significantly lower in SR1 than RE1 $\left(p_{\text {corr }}<0.05\right)$ (supplemental Fig. 1, available at www.jneurosci.org as supplemental material). For the nonsignificant difference in the second time window, see supplemental Figure $3 a$ (available at www. jneurosci.org as supplemental material). Replicating a previous ERP study (Johansson et al., 2007), we also found an enhanced positive slow wave over frontal electrode sites in the selective retrieval condition compared to the reexposure condition (see Supplementary Analysis section, available at www.jneurosci.org as supplemental material).

First versus second cycle

Statistical analyses revealed that SR1 differed from SR2 in the first $\left(0-500 \mathrm{~ms}, p_{\text {corr }}<0.01\right)$ and the second $\left(500-1000 \mathrm{~ms}, p_{\text {corr }}<\right.$ 
$0.05)$ time window, showing a decrease in theta $(5-9 \mathrm{~Hz})$ amplitude from the first to the second cycle (Fig. $3 c$ ). The effect was mainly located over midfrontal and left parietal electrodes (Fig. 3d). See supplemental Figure $3 b$ (available at www. jneurosci.org as supplemental material) for the nonsignificant difference in the third time window. Comparing RE1 with RE2, no significant effects in the theta band were found (see Table 1 and supplemental Fig. $3 c$, available at www.jneurosci.org as supplemental material). A nonparametric correlation $(r=0.59, p<0.01)$ showed that the theta band amplitude in SR1 in the third time window (1000-1500 ms), collapsed over significant electrode positions in the comparison across conditions (SR1 vs RE1), predicted the theta band amplitude in SR2 in the first time window $(0-500 \mathrm{~ms})$, collapsed over significant electrode positions in the comparison across retrieval cycles (SR1 vs SR2).

\section{Interaction analysis}

An interaction analysis with the factors condition (selective retrieval vs reexposure) and cycle (first vs second cycle) was conducted, and revealed a significant effect in the second time window (500-1000 ms, $p_{\text {corr }}<0.05$ ) (Fig. 4a), which was located over midfrontal electrode positions. This effect was due to a pronounced power decrease across cycles in the selective retrieval condition, which was not evident in the reexposure condition (Fig. $4 b$; see Fig. $4 a$, second time window). See supplemental Figure $3 d$ (available at www. jneurosci.org as supplemental material) for nonsignificant differences in the first and third time window.

\section{Brain-behavior relationships}

Following Kuhl et al. (2007), brain-behavior correlations were based upon the suppression score, depicting an individual measure of retrieval-induced forgetting relative to recall performance in the control condition (number of remembered high-frequency items from the control condition minus number of remembered unpracticed, high-frequency items from the selective retrieval condition, divided by number of remembered high-frequency items from the control condition). In the same manner, an enhancement score was established, depicting individual retrieval-induced enhancement relative to recall performance in the control condition (number of remembered practiced, low-frequency items from the selective retrieval condition minus number of remembered low-frequency items from the control condition, divided by number of remembered low-frequency items from the control condition). As electrophysiological measure, we used the differences in theta amplitude across cycles in the second time window (500-1000 ms) of the selective retrieval condition from each participant. As the topographies in Figure $3 d$ suggested, we split electrodes into a frontal and parietal pool. Nonparametric correlations showed that the reduction in theta band amplitude over the frontal pool predicted later forgetting $(r=$ $0.42, p<0.05$ ), whereas the reduction over the parietal pool did not $(r=0.06, p=0.77)$. Enhancement score was not significantly cor- b

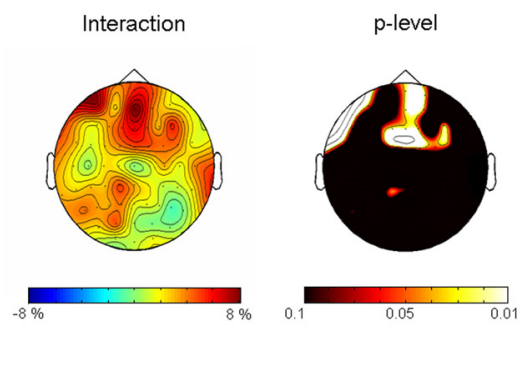

d

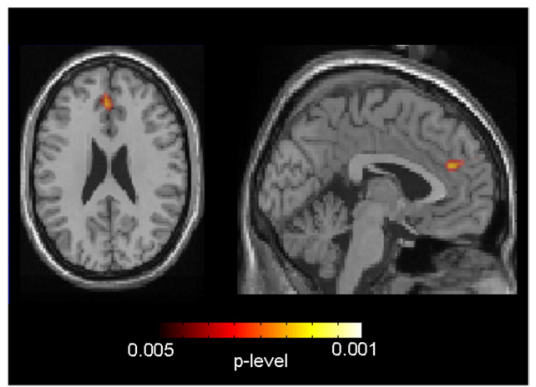

Figure 4. $\quad \boldsymbol{a}$, The time course of the difference in theta amplitude obtained by the interaction analysis (condition $\times$ cycle) is depicted for those electrode positions that showed a significant effect during the second time window (500-1000 ms). Time (Selective retrieval condition are shown. Amplitudes were collapsed over fronta (Beamformer) of the difference in theta amplitude between the first and second cycle of selective retrieval (500-1000 ms) in the anterior cingulate cortex is depicted. Differences for each voxel are plotted by means of $p$ values, obtained by nonparametric Wilcoxon tests.

related with the decrease in theta amplitude, over both the frontal pool and the parietal pool (both $p$ values $>0.10$ ).

To further investigate the brain-behavior relationship, we split the subject sample into a high- and a low-forgetting group, based on the individual suppression scores, and compared their theta band amplitude across cycles in the selective retrieval condition, pooled over frontal electrodes. A two-way ANOVA with the factors group (high vs low forgetters) and cycle (SR1 vs SR2) revealed a significant group $\times$ cycle interaction $\left(F_{(1,22)}=4.99\right.$; $p<0.05$ ), showing that the decrease in theta amplitude from the first to the second cycle during selective retrieval was larger for the high forgetters than the low forgetters (Fig. 4c). In the low-forgetting group, no reliable difference in theta amplitude between SR1 and SR2 was found $\left(t_{(11)}=0.80 ; p=0.44\right)$. No group-by-cycle interaction was found in the reexposure condition $\left(F_{(1,22)}=0.79 ; p=0.38\right)$. For the split into high and low enhancers, based on the individual enhancement score, a two-way ANOVA with factors group (high vs low enhancers) and cycle (SR1 vs SR2) did not reveal a significant interaction $\left(F_{(1,22)}=1.49 ; p=0.23\right)$.

\section{Source localization}

The Beamformer analysis yielded that the decrease in theta amplitude across cycles in the selective retrieval condition was localized to the left anterior cingulate cortex (MNI coordinates: -2 , 40, 24; BA 9/BA 32) (Fig. 4d) and the right occipitoparietal cortex (MNI coordinates: 15, -70, 5; BA 30/BA 23/BA 17) (supplemental Fig. 2, available at www.jneurosci.org as supplemental material). 


\section{Discussion}

The results replicate prior behavioral work (Ciranni and Shimamura, 1999; Anderson et al., 2000; Bäuml and Aslan, 2004), by showing that selective retrieval, but not reexposure, of a subset of studied items leads to (retrieval-induced) forgetting of related unpracticed items. Unlike reexposure, attempting to selectively retrieve a target item creates interference from related items; this interference is resolved by inhibition that suppresses the items' memory representation and leads to later forgetting of the interfering information (Anderson and Spellman, 1995; Spitzer and Bäuml, 2007). As a corollary, the results thus confirm that reexposure can be used as a neural baseline for selective retrieval, to isolate interference and interference resolution (Johansson et al., 2007; Wimber et al., 2009).

In this study, a modification of the retrieval-practice paradigm (Anderson et al., 1994) with repeated cycles of practice in both the selective retrieval and the reexposure condition was used to vary the level of interference within and across conditions. In the selective retrieval condition, interference is assumed to be (partly) resolved by inhibition during the first retrieval cycle, which should result in a lower level of interference in the second cycle; in the reexposure condition, the level of interference should not differ between repeated cycles, as no interference is assumed to arise initially (e.g., Anderson, 2003; Bäuml et al., 2010). The results show exactly this pattern. Selective retrieval induced a higher level of theta activity compared to the reexposure condition, and we found a decrease in theta amplitude from the first to the second cycle in the selective retrieval condition, whereas theta amplitude did not vary from the first to the second cycle in the reexposure condition. These results indicate that theta oscillations follow the suggested dynamics of interference during selective memory retrieval, thus providing a link between brain oscillations and cognitive theory. Here, we examined the hypothesis that theta oscillations reflect the dynamics of interference in selective memory retrieval, thus focusing the analysis on the theta band. With this restriction in analysis, the results leave open the question to what extent the results are frequency specific, an issue that should be addressed in future work.

The results in the retrieval-practice condition agree with Kuhl et al. (2007), who found a reduction in BOLD signal in prefrontal areas across repeated cycles of selective retrieval, indicating that less inhibitory control is necessary when interference has been resolved during initial selective retrieval. Consistent with fMRI studies in which parietal and frontal areas were found to be specifically involved in selective memory retrieval (Kuhl et al., 2007; Wimber et al., 2009), the effects in the theta band were located over frontal and parietal electrode sites. Locating the sources of theta oscillations, we found the difference between the first and second cycle in the selective retrieval condition to be localized in the ACC. This finding agrees with fMRI studies, showing that BOLD signal changes in the ACC predict retrieval-induced forgetting (Kuhl et al., 2007; Wimber et al., 2009), and it is consistent with studies showing a strong contribution of activation in the ACC to frontal theta oscillations (Ishii et al., 1999; Onton et al., 2005). Our results, therefore, link brain oscillations to fMRI findings and suggest that the electrophysiological mechanism underlying activation of ACC during selective memory retrieval may be synchronization of local cell assemblies in the theta rhythm.

The findings of higher activity in the theta band during selective retrieval than reexposure and of a decrease in theta amplitude from the first to the second practice cycle suggest that theta band activity can serve as a neural marker of the dynamics of interfer- ence in selective episodic retrieval. The neural effects of interference resolution during selective retrieval, however, cannot only be seen from a comparison of the first and second retrieval cycle, but can already be seen at the end of the first cycle. Conceivably, in the late time window, theta amplitude during the first cycle predicts the early theta amplitude in the second cycle, which might reflect the residual amount of interference.

Localizing the present theta effect to the ACC may suggest that theta activity reflects both interference detection and its resolution via inhibition in the present experimental task (e.g., Posner et al., 1988). Alternatively, however, ACC activity may mediate interference detection, and not its resolution (Botvinick et al., $1999,2001)$, suggesting that ACC activity only "reads" the state of interference but does not reflect the direct engagement of inhibition as a control mechanism for interference resolution. Recent imaging work supports the latter view, showing that both ACC (interference detection) and prefrontal areas (inhibitory interference resolution) are involved in retrieval-induced forgetting (Kuhl et al., 2007; Wimber et al., 2009). If so, the present theta effect is likely to reflect the effects of interference resolution rather than interference resolution by itself.

Whereas in this study the difference in theta amplitude between the selective retrieval and the reexposure condition is attributed to interference effects, a priori alternative interpretations of the effect may arise. For instance, the theta effect may be due to the presence (as during retrieval) versus absence (as during reexposure) of the retrieval process itself. The fact that theta amplitude decreases across retrieval cycles, however, speaks against such an interpretation. The theta effect might also reflect mental effort, as (actively) retrieving an item from episodic memory might be more effortful than (passively) studying the item again. Following this view, one should expect a correlation between reduction in theta amplitude and enhancement of practiced items; enhancing items should make other (unpracticed) items relatively less competitive, thereby reducing effort for the practiced items on the next practice cycle. Because high enhancers did not show larger decrease in theta amplitude than low enhancers, the results reject such an effort view on the results. Finally, in a similar vein, the repeated cycles of retrieval practice might be contaminated by effects of repetition priming (Grill-Spector et al., 2006). By establishing the reexposure condition as a neural baseline for selective retrieval, however, we were able to control for such an effect. Indeed, the finding of no difference in the theta band between the first and the second cycle of the reexposure condition renders an explanation in terms of repetition priming quite unlikely.

Consistent with our interpretation that the differences in theta amplitude between the selective retrieval and the reexposure condition reflect the dynamics of interference, brain-behavior correlations revealed that the reduction in theta activity over frontal electrodes in the retrieval condition predicted retrieval-induced forgetting. The bigger the decrease from the first to the second cycle in the selective retrieval condition was, the more interfering items were forgotten on the later test. This pattern was evident in the correlation as well as in the behavior-based split of the subject sample. Typically, the finding of no retrieval-induced forgetting on the final recall test is interpreted as the failure to successfully reduce interference in selective memory retrieval, and it is attributed to a deficit in inhibitory function (e.g., Anderson, 2003). Our data, though, suggest an alternative explanation. Whereas high forgetters showed high theta amplitudes during the first cycle, low forgetters showed a low level of theta activity, which did not change from the first to the second cycle. Intriguingly, theta 
activation in the low forgetters generally mimicked the pattern found in the reexposure condition, during which no interference is assumed to arise. Thus, the low forgetters in the present study seem to show a deficit in interference detection rather than a deficit in inhibitory function. The finding mirrors results from a recent fMRI study (Kuhl et al., 2007). In this study, high forgetters showed large initial activation of the ACC, which was reduced on a subsequent retrieval cycle, whereas low forgetters' level of ACC activation was low and remained unchanged during both initial and subsequent cycles of retrieval.

Concerning the ongoing debate on whether retrieval-induced forgetting is inhibitory or noninhibitory in nature (e.g., Anderson, 2003; Jakab and Raaijmakers, 2009), the present results speak in favor of the inhibitory account. Noninhibitory accounts of retrieval-induced forgetting mostly explain the forgetting by assuming that practiced (stronger) items block access to the unpracticed (weaker) items at test, thus suggesting a correlation between enhancement and forgetting. Both behaviorally and neurally, the present results challenge such a view. We found enhancement to be uncorrelated with forgetting, and the theta effect to be predictive for forgetting but not enhancement.

\section{Conclusions}

We showed that (1) activity in the theta band reflects interference during selective memory retrieval, as compared to a noninterference baseline, (2) theta oscillatory activity across repeated cycles of retrieval mimics the hypothesized dynamics of interference in episodic memory retrieval, (3) theta band activity reflecting interference is generated in the ACC, and (4) the reduction in theta band amplitude across cycles predicts successful interference resolution, as indexed by later forgetting of the interfering information. We conclude that activity in the theta frequency range can serve as a neural marker of the dynamics of interference in episodic memory retrieval.

\section{References}

Anderson MC (2003) Rethinking interference theory: executive control and the mechanisms of forgetting. J Mem Lang 49:415-445.

Anderson MC, Neely JH (1996) Interference and inhibition in memory retrieval. In: Memory. Handbook of perception and cognition, Ed 2 (Bjork EL, Bjork RA, eds), pp 237-313. San Diego: Academic.

Anderson MC, Spellman BA (1995) On the status of inhibitory mechanisms in cognition: memory retrieval as a model case. Psychol Rev 102:68-100.

Anderson MC, Bjork RA, Bjork EL (1994) Remembering can cause forgetting: retrieval dynamics in long-term memory. J Exp Psychol Learn Mem Cogn 20:1063-1087.

Anderson MC, Bjork EL, Bjork RA (2000) Retrieval induced forgetting: evidence for a recall-specific mechanism. Psychon Bull Rev 7:522-530.

Bäuml K-H (1998) Strong items get suppressed, weak items do not: the role of item strength in output interference. Psychon Bull Rev 5:459-463.

Bäuml K-H (2008) Inhibitory processes. In: Learning and memory: a comprehensive reference, $\mathrm{Vol}$ 2, Cognitive psychology of memory (Roediger HL 3rd, ed), pp 195-220. Oxford: Elsevier.

Bäuml K-H, Aslan A (2004) Part-list cuing as instructed retrieval inhibition. Mem Cognit 32:610-617.

Bäuml K-H, Pastötter B, Hanslmayr S (2010) Binding and inhibition in episodic memory - cognitive, emotional, and neural processes. Neurosci Biobehav Rev 34:1047-1054.

Blair RC, Karniski W (1993) An alternative method for significance testing of waveform difference potentials. Psychophysiology 30:518-524.
Botvinick M, Nystrom LE, Fissell K, Carter CS, Cohen JD (1999) Conflict monitoring versus selection-for-action in anterior cingulate cortex. $\mathrm{Na}-$ ture 402:179-181.

Botvinick MM, Braver TS, Barch DM, Carter CS, Cohen JD (2001) Conflict monitoring and cognitive control. Psychol Rev 108:624-652.

Cavanagh JF, Cohen MX, Allen JJ (2009) Prelude to and resolution of an error: EEG phase synchrony reveals cognitive control dynamics during action monitoring. J Neurosci 29:98-105.

Ciranni MA, Shimamura AP (1999) Retrieval-induced forgetting in episodic memory. J Exp Psychol Learn Mem Cogn 25:1403-1414.

Gevins A, Smith ME, McEvoy L, Yu D (1997) High-resolution EEG mapping of cortical activation related to working memory: effects of task difficulty, type of processing, and practice. Cereb Cortex 7:374-385.

Grill-Spector K, Henson R, Martin A (2006) Repetition and the brain: neural models of stimulus-specific effects. Trends Cogn Sci 10:14-23.

Gross J, Kujala J, Hamalainen M, Timmermann L, Schnitzler A, Salmelin R (2001) Dynamic imaging of coherent sources: studying neural interactions in the human brain. Proc Natl Acad Sci U S A 98:694-699.

Hanslmayr S, Aslan A, Staudigl T, Klimesch W, Herrmann CS, Bäuml K-H (2007) Prestimulus oscillations predict visual perception performance between and within subjects. Neuroimage 37:1465-1473.

Hanslmayr S, Pastötter B, Bäuml K-H, Gruber S, Wimber M, Klimesch W (2008) The electrophysiological dynamics of interference during the Stroop task. J Cogn Neurosci 20:215-225.

Hanslmayr S, Spitzer B, Bäuml K-H (2009) Brain oscillations dissociate between semantic and nonsemantic encoding of episodic memories. Cereb Cortex 19:1631-1640.

Ishii R, Shinosaki K, Ukai S, Inouye T, Ishihara T, Yoshimine T, Hirabuki N, Asada H, Kihara T, Robinson SE, Takeda M (1999) Medial prefrontal cortex generates frontal midline theta rhythm. Neuroreport 10:675-679.

Jakab E, Raaijmakers JGW (2009) The role of item strength in retrievalinduced forgetting. J Exp Psychol Learn Mem Cogn 35:607-617.

Jensen O, Tesche CD (2002) Frontal theta activity in humans increases with memory load in a working memory task. Eur J Neurosci 15:1395-1399.

Johansson M, Aslan A, Bäuml K-H, Gäbel A, Mecklinger A (2007) When remembering causes forgetting: electrophysiological correlates of retrieval-induced forgetting. Cereb Cortex 17:1335-1341.

Kuhl BA, Dudukovic NM, Kahn I, Wagner AD (2007) Decreased demands on cognitive control reveal the neural processing benefits of forgetting. Nat Neurosci 10:908-914.

Kuhl BA, Kahn I, Dudukovic NM, Wagner AD (2008) Overcoming suppression in order to remember: contributions from anterior cingulate and ventrolateral prefrontal cortex. Cogn Affect Behav Neurosci 8:211-221.

Mannhaupt HR (1983) Reproduktionsnormen für verbale Reaktionen zu 40 geläufigen Kategorien. Sprache und Kognition 4:264-278.

Mecklinger A, Kramer AF, Strayer DL (1992) Event related potentials and EEG components in a semantic memory search task. Psychophysiology 29:104-119.

Onton J, Delorme A, Makeig S (2005) Frontal midline EEG dynamics during working memory. Neuroimage 27:341-356.

Posner MI, Petersen SE, Fox PT, Raichle ME (1988) Localization of cognitive operations in the human brain. Science 240:1627-1631.

Scheithe K, Bäuml K-H (1995) Deutschsprachige Normen für Vertreter von 48 Kategorien. Sprache und Kognition 14:39-43.

Spitzer B, Bäuml K-H (2007) Retrieval-induced forgetting in item recognition: evidence for a reduction in general memory strength. J Exp Psychol Learn Mem Cogn 33:863-875.

Wimber M, Bäuml K-H, Bergström Z, Markopoulos G, Heinze HJ, Richardson-Klavehn A (2008) Neural markers of inhibition in human memory retrieval. J Neurosci 28:13419-13427.

Wimber M, Rutschmann RM, Greenlee MW, Bäuml K-H (2009) Retrieval from episodic memory: neural mechanisms of interference resolution. J Cogn Neurosci 21:538-549. 